\title{
Womit haben wir das verdient? Weniger Geld bei besserer Leistung
}

Frauen verdienen weniger als Männer - das wissen wir aus vielen Untersuchungen. Und trotzdem bleibt in vielen Köpfen das Vorurteil, dieser Unterschied sei auf unterschiedliche Bildung und Ausbildung, typische Berufswahl sowie selbst gewählte Arbeitszeitunterschiede und Erwerbsunterbrechungen zurückzuführen. Auf der Grundlage des Pforzheimer Absolventenpanels können die genannten Faktoren weitgehend kontrolliert werden und es zeigt sich: Die Entgeltunterschiede zwischen Männern und Frauen sind zu einem erheblichen Teil das Ergebnis von Diskriminierung, die schon früh in der Erwerbsbiografie einsetzt.

\section{Einleitung}

Zwischen Frauen und Männern besteht immer noch ein großes Lohngefälle, eine Vielzahl nationaler und internationaler Studien belegt dies (z. B. BMFSFJ 2008; Eurostat 2008; Bispinck et al. 2008; Blau/ Kahn 2006, 2003). Nach einer auf ca. 68.000 Fragebögen basierenden OnlineUmfrage verdienen Frauen über alle Berufe und Altersklassen hinweg im Durchschnitt $22 \%$ weniger als Männer (WSI-Frauenlohnspiegel 2007). Je nach Beruf beträgt der Unterschied zwischen $84 €$ und $825 €$ im Monat. Die Einkommensdifferenz verstärkt sich mit zunehmendem Alter der betrachteten Personen. Sie ist in Deutschland bei Hochschulabsolventinnen und -absolventen und in Führungspositionen besonders groß (BMFSFJ 2009).

Gehaltsunterschiede zwischen Frauen und Männern werden im internationalen Sprachraum als "gender pay gap“ oder "gender wage gap“ bezeichnet. Der „gender pay gap“" wird als das Verhältnis des durchschnittlichen Bruttostundenlohns von Frauen zum durchschnittlichen Stundenlohn von Männern bzw. als der Unterschied zwischen dem Bruttostundenlohn von Frauen und Männern als Prozentsatz des durchschnittlichen Stundenlohns von Männern berechnet (Plantega/Remery 2006). Im europäischen Vergleich lag der "gender pay gap" im Jahr 2005 zwischen $4 \%$ und $25 \%$ (Eurostat 2008). Deutschland nimmt hier seit Jahrzehnten zusammen mit Zypern, Estland und der Slowakei im negativen Sinne eine „Spitzenstellung“ ein.
Andererseits ist in den Jahren 1991 bis 2004 die Frauenerwerbsquote von rund $44 \%$ auf knapp $49 \%$ angestiegen (Wanger 2005). Gleichzeitig stieg der Anteil der Absolventinnen an deutschen Hochschulen (Statistisches Bundesamt 2008a, 2009). Im Jahr 2007 betrug der Anteil der Absolventinnen $52 \%$. Auch der Frauenanteil bei den Promotionen stieg von 2000 bis 2007 von $34 \%$ stetig auf $42 \%$. $^{1}$ Frauen sind demnach bezüglich des formalen Bildungsabschlusses genauso gut qualifiziert wie Männer.

\section{Theorie und Empirie der Entgeltdiskriminierung}

Ein Ansatz zur Erklärung der Verdienstunterschiede von Frauen und Männern ist der einer unterschiedlichen Humankapitalausstattung, die wiederum das Ergebnis verschiedener Präferenzen und Rollenspezialisierungen ist (Becker 1993). Frauen planen demnach aufgrund ihrer Familienorientierung von vornherein einen anderen Berufsweg und investieren z. B. weniger in ihre Ausbildung. Zusätzlich entsteht für Frauen aufgrund der Familienorientierung ein Zeitkonflikt, der auch zulasten des Berufs geht und damit zu einem Verdienstnachteil für Frauen führt (Nivorozhkina/Nivorozhkin 2008). Aufgrund von familiären Erwerbsunterbrechungen können Frauen ihr Humankapital nicht vollständig einbringen (Budig/England 2001). Unterschiedliche Präferenzen und eine Familienorientierung werden über die Entscheidung von Frauen für Berufe, in denen z. B. Teilzeitarbeit möglich ist, über die sogenannte Selbstselektion auch als Erklärungsfaktor für einen Teil der Segregation auf dem Arbeitsmarkt angeführt (Polachek 1981). Es findet so nicht nur eine vertikale Segregation hinsichtlich der Führungsebenen - der Anteil von Frauen in Führungspositionen steigt zwar geringfügig, ist aber gerade in den hohen Positionen immer noch sehr gering (Brader/Lewerenz 2006; Holst 2006; Kleinert 2006) -, sondern auch eine horizontale Segregation hinsichtlich der Art des ausgeführten Berufs statt (z. B. Allmendinger et al. 2006). Andererseits konnte in Studien gezeigt werden, dass Frauen, die bereits zu Beginn ihres Berufslebens eine spätere Unterbrechung ihrer Erwerbstätigkeit planten, sich nicht häufiger als andere Frauen für typische Frauenberufe entschieden

\footnotetext{
1 Erst nach der Promotion sinkt der Frauenantei im universitären Bereich stark. Im Jahr 2007 wurden nur $24 \%$ der Habilitationen von Frauen durchgeführt, der Frauenanteil bei Professuren lag bei $16 \%$, in den höchsten Besoldungsgruppen C4/W3 nur bei $12 \%$ (Statistisches Bundesamt 2008a).
}

Kirsten Wüst, Prof. Dr., lehrt Quantitative Methoden an der Hochschule Pforzheim. Arbeitsschwerpunkte: Finanzrisikomanagement, Simulation, Statistik. e-mail: Kirsten.Wuest@hs-pforzheim.de Brigitte Burkart, Diplom-Psychologin, leitet das Programm zur Förderung der Sozial-, Methoden- und Interkulturellen Kompetenz der Fakultät für Wirtschaft und Recht an der Hochschule Pforzheim und ist stellvertretende Gleichstellungsbeauftragte. Arbeitsschwerpunkte: Personalmanagement, Schlüsselkompetenzen, Gleichstellung e-mail: Brigitte.Burkart@hs-pforzheim.de 
(England, 1982). In Berufen mit höherem Frauenanteil werden dennoch bei gleicher Humankapitalausstattung geringere Löhne gezahlt (vgl. England 1982). Minks (2001) führt an, dass Frauen in technischen und naturwissenschaftlichen Berufen, die nicht frauentypisch sind, deutlich weniger als ihre männlichen Kollegen verdienen.

Die sich aufgrund einer unterschiedlichen Ausbildung ergebenden Lohnunterschiede gelten in der Humankapitaltheorie als legitim, sie sind erklärbar (Blinder 1973; Mincer 1970; Oaxaca 1973). Die vielfach angewandte Oaxaca-Blinder-Zerlegung teilt den Lohnunterschied zwischen Frauen und Männern in den durch die Humankapitalausstattung erklärbaren Anteil und einen Rest auf (Busch/Holst 2008; Blau/Kahn 2006, 2003; Olsen/Walby 2004; Oaxaca 1973). Der nicht durch die Humankapitalausstattung erklärbare Rest wird zumeist unterschiedlichen Arten von Diskriminierung zugeschrieben. Arbeitgeber erwarten aufgrund möglicher Erwerbsunterbrechungen höhere Personalkosten für Frauen und stellen diese daher zu einem geringeren Lohn ein (Becker 1971). Da Arbeitgeber diese Entscheidung aufgrund fehlender Information über die Zukunftspläne einer Arbeitnehmerin, d. h. aufgrund eines Informationsdefizits (Arrow 1974), nicht individuell treffen, spricht Phelps (1972) von einer statistischen Diskriminierung. So sind aber auch Frauen, die in ihrem späteren Leben keine Kinder bekommen werden, von einem niedrigeren Anfangslohnniveau betroffen.

Arbeitgeber betrachten die Geschlechtszugehörigkeit als einfaches Beurteilungskriterium für die in der Zukunft zu erwartende Leistung (Arrow 1974; Phelps 1972), obwohl die Ausbildung gleich gut ist. Dieses gilt besonders auch schon zu Beginn des Berufslebens (Fitzenberger/Kunze 2005). Neben der geringeren Entlohnung von Frauen und Männern in Berufen mit höherem Frauenanteil (England et al. 1988) wird auch innerhalb einer Berufsgruppe die Arbeit von Frauen geringer bezahlt als die von Männern (Achatz et al. 2005), d. h. Frauen werden unmittelbar diskriminiert. Bei der geringeren Berücksichtigung von Frauen für Führungspositionen spielt zudem eine Rolle, dass diejenigen, die die Entscheidung treffen, zumeist Männer sind und Männer in ihrem Netzwerk bevorzugen (McPherson et al. 2001). Neumark (1996) untersuchte so die Chancen von angeblichen Jobsuchenden mit sehr ähnlichen
Unterlagen, die sich in Restaurants mit ihren potenziellen Arbeitgebern trafen. Die Frauen hatten eine bis zu $50 \%$ geringere Chance ein Arbeitsangebot zu erhalten.

Durch statistische und unmittelbare Diskriminierung sind Frauen bei gleichen Qualifikationen und gleichem Arbeitseinsatz gegenüber Männern deutlich benachteiligt. In einer Studie der Wirtschaftsuniversität Wien aus dem Jahr 2005 (Strunk et al. 2005) wurden zur Ausklammerung aller möglicher Effekte „virtuelle Zwillingspärchen" mit jeweils einem männlichen Absolventen und einer Absolventin gebildet, die hinsichtlich der Kriterien Alter, soziale und ökonomische Herkunft, Studienerfolg, Persönlichkeitsstruktur und Einstellung zur Karriere mit Ausnahme des Geschlechts keine signifikanten Unterschiede aufwiesen. Diese „Pärchen“ wurden über einen Zeitraum von zehn Jahren nach dem Hochschulabschluss regelmäßig zu verschiedenen Themen wie Gehaltsentwicklung, Übernahme von Führungsverantwortung und berufliche Zufriedenheit befragt. Sobald ein Teil eines virtuellen „Pärchens" aufgrund von Kinderbetreuung die Erwerbstätigkeit unterbrach, wurde das „Zwillingspaar" aus der Stichprobe eliminiert. Auf diese Art konnte sichergestellt werden, dass der Faktor Kinderbetreuung bei der Gehaltsentwicklung keine Rolle spielte. Die Ergebnisse weisen nach zehnjähriger ununterbrochener Berufstätigkeit einen „gender pay gap“ von 27,7 \% zwischen den „Zwillingen“ aus. Hinz/Gartner (2005) machen für Deutschland bei gleicher Ausbildung, gleichem Beruf und gleicher Berufserfahrung eine Lohndifferenz von $12 \%$ bei Frauen im Vergleich zum Lohn der Männer innerhalb von Betrieben aus. Empirische Studien stellen fest, dass Frauen in Führungspositionen sich in ihrem Humankapital kaum von Männern unterscheiden. Dennoch ist der „gender pay gap “ in Führungspositionen am stärksten ausgeprägt (Holst et al. 2009; Holst 2006; Fitzenberger/Wunderlich 2002).

Psychosoziale Forschungsrichtungen machen einen Teil des "gender pay gap“ an unterschiedlichem Kommunikationsverhalten von Frauen und Männern fest. Dass Frauen der Ansicht sind, dass es mehr auf ihre Leistung ankomme, während Männer über ihre Erfolge reden, wird in der Praxis tatsächlich immer wieder beobachtet (Topf 2005). In verschiedenen Studien wird berichtet, dass Frauen bei Gehaltsverhandlungen zu defensiv auftreten. Doughty (2009) postuliert, dass die Gehaltslücke keine Frage der Diskriminierung von Frauen sei, sondern eine bewusste Entscheidung für einen Lebensstil, in dem die Karriere nicht im Mittelpunkt steht. Demgegenüber berichtet Bischoff (1990, S.185) aus Untersuchungen mit Führungskräften, dass Frauen beim Einstieg in Führungspositionen ,gezwungen (sind), eher Kompromisse beim Gehalt zu machen“. Sie hoffen dann auf späteren Ausgleich, der aber meist nicht erfolgt. Im Hinblick aufGehaltserhöhungen konstatieren Ruppert/Voigt (2009), dass Frauen sogar häufiger um Gehaltserhöhungen anfragen. Dennoch werden Männer bei Gehaltserhöhungen verstärkt berücksichtigt. Zum einen werden sie häufiger als Frauen angesprochen, ohne selbst aktiv werden zu müssen. Zum anderen sind Vollzeitbeschäftigte in Gehaltsverhandlungen häufiger erfolgreich und der Anteil von Männern an den Vollzeitbeschäftigungen ist nach einiger Zeit im Berufsleben deutlich höher. Krell (2010) fasst weitere Diskriminierungsquellen im Hinblick auf variable Vergütungen sowie Zusatz- und Nebenleistungen von Führungskräften zusammen.

\section{Vorgehensweise}

\subsection{KONTROLLE VON EINFLUSS- FAKTOREN}

In der vorliegenden Untersuchung sollen die oben beschriebenen, in der Literatur diskutierten Gründe für weiterbestehende geschlechtsbedingte Gehaltsunterschiede, d. h. eine mögliche unterschiedliche $\mathrm{Hu}-$ mankapitalausstattung von Frauen und Männern aufgrund einer unterschiedlichen Ausbildung, horizontale Segregation aufgrund von Selbstselektion sowie eine geringere Arbeitszeit aufgrund von Teilzeitarbeit und Erwerbsunterbrechungen, durch die Wahl des Datensatzes so weit wie möglich ausgeschlossen werden. Hierzu werden nur Frauen und Männer mit denselben Ausgangsbedingungen in die Studie eingeschlossen. Auf der Grundlage des Pforzheimer Absolventenpanels werden die Einstiegsgehälter von Frauen und Männern verglichen. Alle starten mit demselben Abschluss der Fakultät für Wirtschaft und Recht (Diplom-Betriebswirt) ins Berufsleben, sie haben die gleiche hochwertige Ausbildung und die gleichen Fächer 
studiert. Ihre Humankapitalausstattung ist daher, was die Hochschulausbildung betrifft, identisch. Zwar besteht auch hier die Möglichkeit, dass Absolventinnen und Absolventen sich mit einem Abschluss des gleichen Studienganges auf unterschiedliche Stellen bewerben, doch ist eine typische Wahl von Frauen- oder Männerberufen wesentlich stärker eingeschränkt, da dieselben Studienfächer untersucht werden und eine getrennte Untersuchung nach Fächern vorgenommen wird. In die Untersuchung der Gehaltsunterschiede wurden nur Daten von Vollzeitbeschäftigten einbezogen. Der Faktor Teilzeitarbeit fällt daher in einer statischen Betrachtung als Erklärungsfaktor für die Gehaltsunterschiede zwischen Frauen und Männern ebenfalls heraus. Erwerbsunterbrechungen kommen zu Beginn des Berufslebens noch nicht vor. Gegenstand der Studie ist die berufliche Situation der Absolventinnen und Absolventen der Fakultät für Wirtschaft und Recht der Hochschule Pforzheim unmittelbar nach ihrem Hochschulabschluss. Die Hauptfragestellung dieses Beitrags besteht darin zu überprüfen, ob Unterschiede in den Einstiegsgehältern von Frauen und Männern trotz der Fixierung der genannten Variablen bestehen, und gegebenenfalls Erklärungsfaktoren für diese Ungleichheiten zu finden.

\subsection{DATEN UND METHODEN}

Die Hochschule Pforzheim bietet über 20 Studiengänge in den drei Fakultäten Gestaltung, Technik sowie Wirtschaft und Recht, außerdem zahlreiche Masterstudiengänge an allen drei Fakultäten an. Mit rund 4.500 Studierenden ist Pforzheim eine der größten Fachhochschulen Baden-Württembergs. Die Fakultät für Wirtschaft und Recht stellt mit circa 2.900 Studierenden die größte Einheit dar.

Um die weitere Entwicklung der Hochschulabgänger verfolgen zu können, gibt es seit 1998 in der Fakultät Wirtschaft und Recht regelmäßige Evaluationen das Absolventenpanel. Unmittelbar nach dem Abschluss findet eine Befragung aller Absolventinnen und Absolventen der Fakultät statt. Es liegen hier Daten von 1998 bis 2008 vor. Der Fokus der Befragung nach Abschluss des Studiums liegt auf der rückblickenden Bewertung des Studiums (Relevanz und Qualität der einzelnen Fächer). Für die vorliegende Studie werden aber nur die Daten, die den Berufseinstieg betreffen, sowie von den Studierenden erbrachte Stu-

Tabelle 1: Absolventinnenanteil und Frauenanteil pro Studienfach 1998 bis 2008 - in \% -

\begin{tabular}{lcc} 
Studiengang & $\begin{array}{c}\text { Durchschnittlicher } \\
\text { Absolventinnenanteil }\end{array}$ & $\begin{array}{c}\text { Frauenanteil der } \\
\text { Absolventenbefragung }\end{array}$ \\
\hline Wirtschaftsinformatik & 13,5 & 16,0 \\
Logistik & 30,2 & 31,4 \\
Controlling & 38,0 & 37,2 \\
Steuern & 43,0 & 47,4 \\
Marketing & 46,5 & 44,1 \\
Wirtschaftsrecht & 53,4 & 61,7 \\
Marktforschung & 54,0 & 57,4 \\
Werbung & 60,9 & 62,1 \\
International Business & 69,4 & 70,4 \\
Personalmanagement & 72,1 & 70,7 \\
\hline \multirow{2}{*}{ Quelle: Pforzheimer Absolventenpanel; Berechnungen der Autorinnen. } & WSI MITTEILUNGEN \\
\hline \hline
\end{tabular}

dienleistungen verwendet. Es wird erfasst, ob bereits eine Zusage für eine Stelle vorliegt sowie gegebenenfalls das vereinbarte Bruttojahresanfangsgehalt. Ferner wird ausgewertet: die Abschlussnote; ob ein Auslandsaufenthalt ins Studium integriert wurde; das Engagement in studentischen Organisationen; die Zeit, die in Praktika verbracht wurde. Des Weiteren gehen der Schulabschluss, die berufliche Vorausbildung, die Erwerbstätigkeit während des Studiums und die Mobilität der Studierenden, gemessen daran, ob eine Stellensuche bundesweit oder regional erfolgte, in die Untersuchung ein. Da es das Ziel der Untersuchung ist, die Variablen Ausbildung, Erwerbsunterbrechungen, etc. für die Geschlechter konstant zu halten, werden die Gehaltsdaten einer Folgebefragung nach mehreren Berufsjahren nicht in die Studie aufgenommen. Aus der Folgebefragung wird nur die Beurteilung gegebener Kriterien, um im Beruf erfolgreich zu sein und hier speziell des Kriteriums „das richtige Geschlecht zu haben“, ausgewertet.

Bei der Durchführung statistischer Tests werden zweiseitige $p$-Werte berichtet. Ein $\mathrm{p}$-Wert $\mathrm{p}<0,05$ gilt als signifikant. Es erfolgt eine explorative Auswertung, daher wurden keine Anpassungen für multiple Tests vorgenommen.

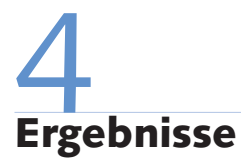

\subsection{STRUKTUR DER ABSOLVENTEN- GRUPPE}

An der Fakultät für Wirtschaft und Recht waren im Sommersemester 2008 insgesamt 2.617 Studierende immatrikuliert, der Frauenanteil betrug 52,8 \%. Der Anteil der weiblichen Studierenden nahm von 1998 mit 42,7 \% bis 2007 mit 56,5\% stetig zu (leichter Rückgang 2008). Der Frauenanteil liegt dabei leicht über dem Durchschnitt der BWL-Studienfächer an deutschen Hochschulen von 46,6 \% (Statistisches Bundesamt 2008b). Dieses liegt sicherlich an der hohen Zahl von Studiengängen mit hohen Frauenanteilen wie den Studiengängen Werbung und Personalmanagement.

Aus der Verteilung der Studierenden auf die Studiengänge lässt sich durchaus eine Präferenz der weiblichen Studierenden für bestimmte Studienfächer ausmachen. Insbesondere überwiegen Frauen in den Fächern mit hohem Kommunikationsanteil, wie z. B. Werbung, International Business und Personalmanagement. Sie sind in den eher "technisch“ ausgerichteten Fächern der Wirtschaftswissenschaften wie Wirtschaftsinformatik und Logistik deutlich unterrepräsentiert.

An der Befragung nahmen von 1998 bis 20083.045 Personen teil, 49,9\% waren männlich, 50,1 \% weiblich. Die Anteile an Frauen pro Studiengang, die an der Befragung teilgenommen hatten, sind in Tabelle 1 dem Durchschnitt der Frauenanteile an allen Absolventinnen und Absolventen während des Studienzeitraums gegenübergestellt. Bis auf das Fach Wirtschaftsrecht, ${ }^{2}$

2 Der Studiengang Wirtschaftsrecht besteht in Pforzheim erst seit 1996, sodass die ersten Absolventinnen und Absolventen erst im Wintersemester 2000/2001 ihr Studium beendeten. Die absolute Zahl der Fragebögen aus dem Studiengang Wirtschaftsrecht liegt daher mit 133 deutlich unter denen der anderen Studiengänge, die zwischen 213 und 454 schwanken. Die Varianz ist entsprechend im Studiengang Wirtschaftsrecht höher. 
Tabelle 2: Durchschnittliche Bruttojahreseinkommen (Einstiegsgehalt)

\begin{tabular}{|c|c|c|c|c|c|}
\hline \multirow[b]{2}{*}{ Studiengang } & \multicolumn{2}{|c|}{ in Euro } & \multicolumn{3}{|c|}{ in $\%$} \\
\hline & männlich & weiblich & Differenz & $\begin{array}{l}\text { von } \\
\text { männlich }\end{array}$ & p-Wert \\
\hline $\begin{array}{l}\text { Wirtschaftsinformatik } \\
(\mathrm{N}=104)\end{array}$ & $\begin{array}{l}40.084,27 \\
(\mathrm{~N}=89)\end{array}$ & $\begin{array}{l}37.166,67 \\
(\mathrm{~N}=15)\end{array}$ & $2.917,60$ & 7,3 & 0,152 \\
\hline $\begin{array}{l}\text { Logistik } \\
(\mathrm{N}=133)\end{array}$ & $\begin{array}{c}38.451,09 \\
(\mathrm{~N}=92)\end{array}$ & $\begin{array}{l}37.012,20 \\
(\mathrm{~N}=41)\end{array}$ & $1.438,89$ & 3,7 & 0,288 \\
\hline $\begin{array}{l}\text { Controlling } \\
(\mathrm{N}=203)\end{array}$ & $\begin{array}{l}39.855,07 \\
(\mathrm{~N}=138)\end{array}$ & $\begin{array}{c}37.538,46 \\
(\mathrm{~N}=65)\end{array}$ & $2.316,61$ & 5,8 & $0,022^{*}$ \\
\hline $\begin{array}{l}\text { Steuern } \\
(\mathrm{N}=209)\end{array}$ & $\begin{array}{c}36.194,69 \\
(\mathrm{~N}=113)\end{array}$ & $\begin{array}{c}35.104,17 \\
(\mathrm{~N}=96)\end{array}$ & $1.090,52$ & 3,0 & $0,042^{*}$ \\
\hline $\begin{array}{l}\text { Marketing } \\
(\mathrm{N}=160)\end{array}$ & $\begin{array}{c}38.906,25 \\
(\mathrm{~N}=96)\end{array}$ & $\begin{array}{c}35.976,56 \\
(\mathrm{~N}=64)\end{array}$ & $2.929,69$ & 7,5 & $0,009 * *$ \\
\hline $\begin{array}{l}\text { Wirtschaftsrecht } \\
(\mathrm{N}=55)\end{array}$ & $\begin{array}{l}41.195,65 \\
(\mathrm{~N}=23)\end{array}$ & $\begin{array}{c}38.750,00 \\
(\mathrm{~N}=32)\end{array}$ & $2.445,65$ & 5,9 & 0,113 \\
\hline $\begin{array}{l}\text { Marktforschung } \\
(\mathrm{N}=81)\end{array}$ & $\begin{array}{l}35.426,83 \\
(\mathrm{~N}=41)\end{array}$ & $\begin{array}{l}34.062,50 \\
(\mathrm{~N}=40)\end{array}$ & $1.364,33$ & 3,9 & 0,091 \\
\hline $\begin{array}{l}\text { Werbung } \\
(\mathrm{N}=166)\end{array}$ & $\begin{array}{l}35.188,68 \\
(N=53)\end{array}$ & $\begin{array}{c}32.101,77 \\
(\mathrm{~N}=113)\end{array}$ & $3.507,26$ & 10,0 & $0,017^{*}$ \\
\hline $\begin{array}{l}\text { International Business } \\
(\mathrm{N}=101)\end{array}$ & $\begin{array}{l}39.375,00 \\
(\mathrm{~N}=32)\end{array}$ & $\begin{array}{c}36.666,67 \\
(\mathrm{~N}=69)\end{array}$ & $2.708,33$ & 6,9 & 0,061 \\
\hline $\begin{array}{l}\text { Personalmanagement } \\
(\mathrm{N}=134)\end{array}$ & $\begin{array}{c}36.937,50 \\
(\mathrm{~N}=40)\end{array}$ & $\begin{array}{l}34.627,66 \\
(\mathrm{~N}=94)\end{array}$ & $2.309,84$ & 6,3 & 0,195 \\
\hline $\begin{array}{l}\text { Insgesamt } \\
(\mathrm{N}=1346)\end{array}$ & $\begin{array}{c}38.260,11 \\
(\mathrm{~N}=717)\end{array}$ & $\begin{array}{c}35.298,09 \\
(\mathrm{~N}=629)\end{array}$ & $3.034,64$ & 7,9 & $<0,001 * *$ \\
\hline
\end{tabular}

bei dem eine Abweichung um 8,3\% nach oben besteht, liegen die Werte sehr dicht beieinander, sodass die Daten der Absolventenstudie als repräsentativ für die entsprechenden Studiengänge angesehen werden können.

Zum Zeitpunkt der Befragung hatten $52,1 \%$ der männlichen, aber nur 44,4\% der weiblichen Studenten bereits eine $\mathrm{Zu}$ sage für eine Stelle $(\mathrm{p}<0,001)$.

\section{SCHULISCHE UND BERUFLICHE VORBILDUNG}

Die schulische Ausbildung unterscheidet sich signifikant $(\mathrm{p}<0,001) \quad[\mathrm{p}<0,001]^{3}$ zwischen den Geschlechtern. Während die Studentinnen zu 76,7 \% [76,5 \%] von Gymnasien und Fachgymnasien zur Hochschule wechseln, ist dieses bei den Studenten nur zu 63,2 \% [63,1 \%] der Fall. Einen Fachoberschul-, Kolleg- oder Berufskollegabschluss bringen 33,4\% [32,7\%] der männlichen Studienanfänger, aber nur $20,9 \%$ [21,4 \%] der Studienanfängerinnen mit.

Zusätzlich unterscheidet sich die berufliche Vorbildung der Studierenden signifikant. 55,1 \% [57,6\%] der Absolventen, aber nur 47,4 \% [52,7\%] der Absolventinnen hatten bereits eine Lehre abgeschlossen $(\mathrm{p}<0,001)[\mathrm{p}=0,026]$. Eine schulische Berufsausbildung hatten 5,1\% [5,1\%] der Frauen, aber nur 2,8 \% [2,5\%] der Männer abgeschlossen.

\section{STUDIENLEISTUNGEN}

Studentinnen haben mit einer durchschnittlichen Abschlussnote von 2,13 [2,10] gegenüber Studenten mit einer Durchschnittsabschlussnote von 2,24 [2,21] signifikant $(\mathrm{p}<0,001)[\mathrm{p}<0,001]$ bessere Noten. Die kumulierten Häufigkeiten der Abschlussnoten der Frauen liegen durchweg über denjenigen der Männer. So konnten z. B. 48,6 \% [51,1 \%] der Frauen eine Abschlussnote von 2,0 oder besser aufweisen, während dies bei den Männern nur bei 37,1 \% [38,5 \%] der Fall war.

Mit 44,8 \% [43,7 \%] gegenüber 37,4 \% $[39,1 \%]$ hatten die Studentinnen häufiger einen Auslandsaufenthalt in ihr Studium integriert $(p<0,001)[p=0,077]$. Sie engagierten sich mit $37,2 \%$ [37,3\%] auch etwas mehr in studentischen Organisationen als ihre männlichen Kollegen mit 34,0 \% $[33,6 \%](p=0,068)[p=0,141]$.

\section{ERWERBSTÄTIGKEIT WÄHREND DES STUDIUMS}

Die Erwerbstätigkeit von Studenten mit $83,5 \%[81,0 \%]$ und Studentinnen mit $84,0 \%[81,3 \%]$ unterscheidet sich während des Studiums nicht signifikant $(\mathrm{p}=0,803)$ $[p=0,878]$. Ebenso ist die absolvierte Zeit in Praktika zwischen den Geschlechtern nicht signifikant verschieden $(p=0,575)$ $[p=0,448]$. Bei Studentinnen liegt diese Studenten bei 10,72 [10,99] Monaten. im Mittel bei 10,82 [10,76] Monaten, bei

\section{MOBILITÄT}

Bei der Suche nach einer gut bezahlten Stelle ist die Mobilität ein entscheidender Faktor. Die Mobilität wird anhand der Frage gemessen, ob die Stellensuche bundesweit erfolgte oder nicht. Während 40,5\% [34,9\%] der Absolventen eine bundesweite Joborientierung angaben, waren dieses bei den Absolventinnen nur $34,9 \%$ [30,6 \%]. Der Unterschied ist signifikant für die Gesamtheit aller Befragten $(\mathrm{p}<0,001)$, allerdings nur grenzwertig signifikant für die Personen, die bei der Befragung bereits eine Stellenzusage hatten $[\mathrm{p}=0,086]$.

\subsection{GEHALT BEIM EINSTIEG IN DEN ARBEITSMARKT}

Tabelle 2 zeigt die durchschnittlichen Einstiegsbruttojahresgehälter der Studierenden, die bei ihrem Abschluss bereits eine Zusage für einen Arbeitsplatz hatten und so ihr Einstiegsgehalt genau angeben konnten.

Über alle Studiengänge hinweg verdienten Männer im Mittel pro Jahr rund $3.000 €$ mehr als ihre weiblichen Mitbewerberinnen $(p<0,001)$. Dieser Effekt bleibt auch bei einer Differenzierung nach den Studiengängen erhalten. Zwar unterscheiden sich die Einstiegsgehälter je nach Studienabschluss deutlich $(\mathrm{p}<0,001)$, jedoch liegen in allen Studiengängen die Einstiegsgehälter der Männer über denjenigen der Frauen. Am deutlichsten sind die Unterschiede im Bereich Werbung $(p=0,017)$ mit einem durchschnittlich um $10 \%$, das heißt circa $3.500 €$, höheren Jahreseinkommen der Männer sowie im Bereich Marketing ( $\mathrm{p}=0,009)$, in dem das Jahreseinkommen der Männer durchschnittlich um 7,5\% höher ist als dasjenige der Frauen. Dieses sind auch Studiengänge, in denen der Frauenanteil groß ist.

Zusätzlich wurde eine Regression der logarithmierten Bruttojahreseinkommen auf die in Tabelle 3 aufgeführten Variablen durchgeführt. Die Regression ergab einen signifikanten Einfluss des Geschlechts und der Abschlussnote (jeweils $\mathrm{p}<0,001$ ), eines

\footnotetext{
3 In eckigen Klammern [] sind bei dieser und den folgenden Auswertungen die Werte angegeben, die sich für die Studierenden, die bereits eine Zusage erhalten haben, ergeben.
} 
Auslandsaufenthalts während des Studiums $(\mathrm{p}=0,002)$ und des Abschlussjahres $(p=0,001)$. Ferner verdienten Absolventen und Absolventinnen besser, wenn sie eine abgeschlossene Lehre aufweisen konnten $(p<0,001)$ oder wenn sie ihre Stellensuche auf das gesamte Bundesgebiet ausgeweitet hatten $(p=0,040)$. Aus der Regression ergibt sich ein um 8,7\% geringerer Durchschnittsverdienst für Frauen aufgrund ihres Geschlechts. Die Differenz zum oben aufgeführten niedrigeren wahren Unterschied der Durchschnittsgehälter lässt sich unter anderem durch die besseren Abschlussnoten und die höhere Bereitschaft zu Auslandsaufenthalten bei Frauen erklären. Mit einer Verschlechterung der Abschlussnote um eine ganze Note sinkt das Anfangsgehalt um 6,5\%, bei einem in das Studium integrierten Auslandsaufenthalt in Form eines Praktikums, Auslandsstudiums oder Sprachkurses steigt es um 4,1\%. Hätten die Frauen nicht die besseren Noten und würden häufiger während ihres Studiums ins Ausland gehen, so wäre der Unterschied zwischen den Einstiegsgehältern dementsprechend noch höher. Andererseits steigt das durchschnittliche Anfangsgehalt auch an, wenn vor dem Studium eine Lehre abgeschlossen wurde. Eine abgeschlossene Lehre lässt einen Anstieg von 4,8 \% beim Bruttojahresgehalt erwarten. Von diesem Effekt profitieren vor allem die männlichen Studienabgänger, da sie eine leicht höhere Häufigkeit von abgeschlossenen Lehren aufweisen können. Ebenso gaben die männlichen Absolventen eine zwar nicht signifikante, aber doch höhere Mobilität an. Ihre Stellensuche dehnten sie - stärker als ihre Kolleginnen - bundesweit aus. Bei einer höheren Mobilität steigt das zu erwartende Gehalt ebenfalls leicht, d.h. um $2,7 \%$, an.

Von 1998 an stieg das durchschnittliche Einstiegsgehalt mit jedem Jahr im Mittel um $0,7 \%$. Keinen signifikanten Einfluss auf das Bruttojahresanfangsgehalt hatten die Länge der durchgeführten Praktika, die Erwerbstätigkeit während des Studiums und der Schulabschluss.

In zwei zusätzlichen Regressionen ${ }^{4}$ sind wir dem Effekt der Studiengänge auf Entgelt und Abschlussnoten vertieft nachgegangen. Nimmt man die Studiengänge als Dummy-Variable auf - als Referenz gilt der Studiengang Werbung - so gibt es deutliche Unterschiede der Bruttojahresgehälter zwischen den Fächern. Berufsanfängerinnen und -anfänger aller anderen
Tabelle 3: Was beeinflusst Gehaltsunterschiede? Log-lineare Regression der Anfangsgehälter von Studienabsolventen

\begin{tabular}{|c|c|c|c|c|}
\hline & Beta & $\begin{array}{c}\text { Relative } \\
\text { Lohndifferenz } \\
\text { [Exp(Beta)-1] } \\
* 100 \text { in } \%\end{array}$ & $\begin{array}{l}\text { Standard- } \\
\text { fehler }\end{array}$ & p-Wert \\
\hline Konstante & 10,586 & & 0,048 & $<0,001 * *$ \\
\hline Geschlecht & $-0,091$ & $-8,7$ & 0,012 & $<0,001 * *$ \\
\hline Auslandsaufenthalt & 0,040 & 4,1 & 0,013 & $0,002 * *$ \\
\hline Note & $-0,067$ & $-6,5$ & 0,016 & $<0,001 * *$ \\
\hline Abschlussjahr & 0,007 & 0,7 & 0,002 & $0,001 * *$ \\
\hline Joborientierung bundesweit & 0,026 & 2,7 & 0,013 & $0,040^{*}$ \\
\hline Praktikantenzeit & 0,001 & 0,1 & 0,001 & 0,535 \\
\hline Erwerbstätigkeit während des Studiums & 0,004 & 0,4 & 0,016 & 0,790 \\
\hline Schulabschluss: Gymnasium & $-0,001$ & $-0,1$ & 0,014 & 0,960 \\
\hline abgeschlossene Lehre & 0,047 & 4,8 & 0,015 & $0,001 * *$ \\
\hline abgeschlossene schul. Berufsausbildung & 0,028 & 2,8 & 0,034 & 0,412 \\
\hline
\end{tabular}

Studiengänge verdienen - zum Teil bis zu durchschnittlich $20 \%$ - mehr als diejenigen des Studiengangs Werbung. Der Effekt des Geschlechts geht jedoch nicht verloren. Auch bei Herausrechnen des Studiengangeffekts, d. h. innerhalb desselben Studiengangs, verdienen die weiblichen Absolventen noch 6,4 \% weniger als ihre männlichen Kollegen.

Die Ergebnisse einer linearen Regression auf die Abschlussnoten, bei der neben den Variablen Geschlecht, Auslandsaufenthalt, etc. auch die Studiengänge aufgenommen wurden - wiederum mit dem Studiengang Werbung als Referenz - zeigt: Die Noten der Studierenden sind - zum Teil sogar deutlich - schlechter als diejenigen der Studierenden des Studiengangs Werbung. Dennoch bleibt auch hier der Effekt des Geschlechtes bestehen. Frauen haben im Schnitt innerhalb der Studiengänge eine um 0,08 bessere Note als Männer $(\mathrm{p}<0,001)$; Studierende, die einen Auslandsaufenthalt in ihr Studium integriert hatten, haben Noten, die durchschnittlich um 0,149 besser sind $(\mathrm{p}<0,001)$. Diese Unterschiede scheinen sich hingegen nicht unmittelbar auf die Bruttoanfangsgehälter zu übertragen. Sind die Noten der Werbungsabsolventinnen und -absolventen, die an der Studie teilgenommen haben, auch mit am besten, so waren ihre Bruttoanfangsgehälter doch die niedrigsten. Das Fach, in dem der Studienabschluss erlangt wurde, spielt für die Höhe des Anfangsgehaltes eine deutlich wichtigere Rolle als die Abschlussnote.

\section{Diskussion}

Zwischen Frauen und Männern bestehen noch immer große Verdienstunterschiede. Die Lücke zwischen den Gehältern klafft zwar mit zunehmender Dauer des Berufslebens weiter auseinander, besteht aber bereits beim Eintritt in den Arbeitsmarkt. Unsere Studie belegt diese bekannte Tatsache noch einmal eindringlich. Die dargestellten Ergebnisse zeigen für die Gruppe der Absolventinnen der Fakultät Wirtschaft und Recht der Hochschule Pforzheim bereits unmittelbar nach Abschluss ihres Studiums trotz gleicher qualitativ hochwertiger Ausbildung und sogar besserer Studienleistungen der Frauen schon beim Einstieg in den Beruf Einkommensdifferenzen von durchschnittlich $3.000 €$ im Jahr (7,9 \% des Jahreseinkommens der Männer). Dieser Gehaltsunterschied erstreckt sich in unterschiedlichem Ausmaß über alle Studiengänge und ist damit nicht darauf zurückzuführen, dass Frauen andere Fächerpräferenzen haben und sich von vornherein aufStellen bewerben, deren geringere Bezahlung auf den akademischen Abschluss zurückzuführen wäre. Der „gender pay gap“ erhöht sich nach vier bis neun Jahren im Beruf auch bei den Pforzheimer Absolventinnen und Absolventen

\footnotetext{
4 Die Ergebnistabellen dieser Regressionen stellen die Autorinnen auf Nachfrage gerne zur Verfügung, ebenso wie eine grafische Darstellung der durchschnittlichen Einstiegsgehälter im Zeitverlauf.
} 
bereits auf ca. 20 \%. Diese Ergebnisse werden hier aber nicht näher dargestellt, da, wie bereits erläutert, eine Konzentration auf die Einstiegsgehälter die Homogenität der betrachteten Arbeitnehmerinnen und Arbeitnehmer fördert.

Zum Zeitpunkt der Befragung unmittelbar nach Studienabschluss hatten signifikant mehr Männer als Frauen bereits eine Zusage für eine Stelle. Zwar ist es möglich, dass Frauen auf eine besser bezahlte Stelle warten, die sie erst nach dem Befragungstermin in der Absolventenstudie erhalten. Dieses erscheint jedoch nicht sehr wahrscheinlich. Das Argument wird so auch häufig umgekehrt als Erklärung für die höheren Gehälter der Männer angebracht (vgl. z. B. Spiegel-Online 2007). Männer würden eher auf die perfekte Stelle warten, die dann entsprechend bezahlt wird, auch wenn dieses bedeutet, eine Zeit lang ohne Stelle zu sein. Frauen hingegen würden oft auch Einstiegsstellen annehmen, für die sie eigentlich zu gut qualifiziert sind. Unsere Ergebnisse spiegeln diese Resultate nicht wieder, die Männer haben häufiger bereits früh eine Stellenzusage. Möglicherweise ist es hier eher so, dass Männer früher eine Stelle aufweisen können, da sie bereits nach den ersten Verhandlungen mit dem angebotenen Gehalt zufrieden sind. Über die Art der Verhandlungsführung erlauben die Daten leider keine Aussage.

Natürlich ist es möglich, dass in den Daten eine Verzerrung hinsichtlich der Teilnahme an der Studie in dem Sinne besteht, dass Männer nur dann antworten, wenn sie eine gut bezahlte Stelle erhalten haben, Frauen hingegen auch dann pflichtbewusst den Fragebogen ausfüllen, wenn sie eine geringer dotierte Stelle erhalten haben. Wäre es so, könnte ein Teil des gefundenen Lohnunterschiedes zwischen Frauen und Männern erklärt werden. Diese Frage ist aufgrund der Daten nicht zu entscheiden.

\subsection{BERUFSWAHL UND ÜBERNAHME VON FÜHRUNGSVERANTWORTUNG}

Die Erklärung des „gender pay gap“ durch die horizontale Segregation, d. h. die Entscheidung von Frauen für Branchen und Berufe, z. B. in der Lehre oder Pflege, in denen in der Regel weniger bezahlt wird als in anderen Bereichen, wie z. B. in technischen Berufen, greift hier zu kurz. Dieser Faktor wurde im Pforzheimer Absolventenpanel weitestgehend ausgeschlossen, da die Gehaltsunterschiede innerhalb der verschiedenen Studiengänge betrachtet werden. Zwar können sich auch Frauen ein und desselben Studienganges auf unterschiedliche Tätigkeiten bewerben (s.u.), doch sind die Richtung und so eventuell auch die Branche durch den Studienabschluss größtenteils vorgegeben.

Auch die vertikale Segregation durch den geringeren Anteil von Frauen in Führungspositionen kommt als Erklärungsfaktor für die Gehaltsdifferenzen kaum infrage, da die Einstiegspositionen von Absolventen in den seltensten Fällen Führungsfunktionen beinhalten. Interessant ist es aber, den weiteren Karriereverlauf der Absolventen zu betrachten. Aus Verlaufsstudien für die Pforzheimer Absolventinnen und Absolventen ergibt sich, dass zum Zeitpunkt der Folgebefragung (vier bis neun Jahre nach dem Abschluss) 36,6 \% der Männer Führungsverantwortung übernommen hatten, während dieses nur für 25,6\% der Frauen galt $(p=0,012)$. Da die Gehälter mit zunehmender Führungsverantwortung steigen, liegt in der Übernahme von Führungsaufgaben ein Schlüsselfaktor für die Gehaltsentwicklung, der so auch zumindest einen Teil der Einkommensunterschiede zwischen Frauen und Männern erklären kann.

\subsection{FAMILIÄR BEDINGTE ERWERBS- UNTERBRECHUNGEN UND TEILZEIT- ARBEIT}

Die Faktoren Erwerbsunterbrechung und Teilzeit können als Erklärungsfaktoren in unserer Studie beim Berufseinstieg ausgeschlossen werden, da nur Arbeitsverhältnisse mit Vollzeitbeschäftigung in die Auswertung einbezogen wurden und die Absolventinnen und Absolventen bei ihrem Studienabschluss noch keine Erwerbsunterbrechung aufgewiesen haben.

Dennoch ist es möglich, dass Frauen sich bereits beim Berufseinstieg bewusst bei Firmen und für Tätigkeiten bewerben, die zukünftig mit Familie vereinbar sind. Manche Unternehmen sind für ihre guten Wiedereinstiegsmöglichkeiten nach einer Elternzeit bekannt, hier ist eine Selbstselektion im Hinblick auf die spätere Vereinbarkeit des Berufs mit Familie denkbar. Zusätzlich kann es sein, dass beim Berufseinstieg Teilzeit zwar noch nicht in Anspruch genommen wird, die Tätigkeit aber eine spätere Teilzeit ermöglichen würde und von Frauen Teilzeit stärker bereits beim Berufseinstieg antizipiert wird als von Männern. Aus den gleichen Gründen ist es vorstellbar, dass Frauen Stellen, die mit mehr Überstunden und Dienstreisen verbunden sind, stärker als Männer meiden. Diese Faktoren können so trotz gleicher Ausgangsbedingungen im Sinne einer Selbstselektion einen Teil der Gehaltsunterschiede zu Beginn des Berufslebens erklären.

\subsection{GEHALTSVERHANDLUNGEN UND MOBILITÄT}

Die unterschiedlichen Präferenzen von Frauen in Bezug auf eine stärkere Ausrichtung auf die Vereinbarkeit von Beruf und Familie kommen heute erst nach der Ausbildung bzw. dem Studium zum Tragen. Dies steht im Einklang mit sehr hohen Studienanfängerinnenzahlen. Frauen ist ihre Hochschulausbildung heute wichtig; sie wird nicht mehr aufgrund der Planung eines späteren Familienlebens als „nicht lohnend" angesehen, wie es in früheren Zeiten der Fall war. Dennoch, betrachtet man den universitären Bereich, so sinkt der Anteil der Frauen nach der Promotion. Habilitation und ein Hinarbeiten auf eine Professur sind immer noch schwer mit einem Familienleben zu vereinbaren. Auch bei Gehaltsverhandlungen in der Privatwirtschaft kann es so sein, dass Frauen bewusst weniger Gehalt fordern oder sich trotz gleicher Ausbildung auf andere Stellen als Männer bewerben, um eine Vereinbarkeit von Familie und Beruf zu erzielen (s.o.). Das Pforzheimer Absolventenpanel erlaubt hier keine Rückschlüsse. Die Untersuchung dieser Fragestellung mit einem erweiterten Panelfragebogen ist Bestandteil weiterer Analysen.

Ein Grund, warum Frauen bei Gehaltsverhandlungen nicht die gleiche Hartnäckigkeit zeigen wie Männer, kann so auch in ihrer Lebensplanung liegen. Viele Frauen streben eine Vereinbarkeit von Familie und Beruf an. Ein höheres Gehalt ist für Frauen emotional und faktisch verbunden mit bedingungslosem Einsatz für das Unternehmen. Damit würden sie die Balance riskieren - ihre eigene sowie die für die Familie (BMFSFJ 2009, S.9). Obwohl Frauen also heute aufgrund ihrer Präferenzen häufig keine geringere schulische bzw. universitäre Ausbildung mehr haben, kann das von Becker (1993) formulierte Kosten-Nutzen-Kalkül dennoch einen Teil des „gender pay gap“ erklären. 
Die Daten aus unserer Studie belegen, dass das zu erwartende Einstiegsgehalt der Absolventinnen und Absolventen mit der Bereitschaft steigt, sich bundesweit um eine Stelle zu bewerben. Diese Bereitschaft ist bei den Männern in der vorliegenden Untersuchung höher ausgeprägt als bei den Frauen. Damit lässt sich aber nur ein kleiner Teil der Gehaltsunterschiede erklären.

\subsection{DISKRIMINIERUNG}

Aufgrund der Homogenität des Studienkollektivs der betroffenen Berufsanfängerinnen und Berufsanfänger sind die unmittelbare und statistische Diskriminierung von Frauen beim Berufseinstieg als Erklärungsfaktor für ihr durchschnittlich geringeres Gehalt in Betracht zu ziehen.

Wie bereits in anderen Studien untersucht (Neumark 1996), konnten auch in unserer Studie Frauen zum gleichen Befragungszeitpunkt deutlich weniger häufig eine Stelle vorweisen. Da Frauen andererseits mehr Engagement bei Auslandssemestern und in studentischen Initiativen aufweisen, ist es unwahrscheinlich, dass dieses daran liegt, dass sie sich weniger um eine Stelle bemühen. Es ist daher anzunehmen, dass eine statistische Diskriminierung vorliegt. Demnach unterstellen Arbeitgeber den Absolventinnen - aufgrund von statistischen Durchschnittserwartungen -, dass Erwerbsunterbrechungen oder -reduzierungen einzuplanen sind. In diesem Fall werden männliche Bewerber trotz gleicher Qualifikationen den weiblichen Bewerbern vorgezogen.

Bezüglich des Lohngefälles bleibt als ein Faktor zu vermuten, dass Frauen bei gleicher Qualifikation schlichtweg weniger Einstiegsgehalt angeboten wird, sei es aufgrund erwarteter zukünftiger Erwerbsausfälle wegen Kindererziehung oder aus anderen Gründen. Dieses stellt eine unmittelbare Diskriminierung dar.

Während Schülerinnen und Studentinnen sich gegenüber ihren männlichen Kollegen gleichberechtigt fühlen und nicht die Erfahrung einer Diskriminierung ma- chen, wandelt sich dieses Bewusstsein mit zunehmender Berufserfahrung. Nach einer gewissen Zeit im Berufsleben fühlen Frauen sich durch die noch immer existierende Diskriminierung im Beruf aufgrund ihres Geschlechts so auch stark benachteiligt, während Männer eher nicht realisieren, dass es eine Benachteiligung von Frauen in der Arbeitswelt noch gibt. Das „richtige Geschlecht" zu haben, beurteilen nach vier bis neun Jahren im Beruf 28,5\% der befragten Pforzheimer Absolventinnen als „wichtig“ oder „sehr wichtig“ für das berufliche Fortkommen, während nur 5,9 \% der Absolventen dieser Ansicht sind $(\mathrm{p}<0,001)$.

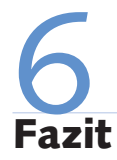

Frauen haben heute die gleiche gute Ausbildung wie Männer. Dennoch verdienen sie nach wie vor weniger. Unsere Studie zeigt, dass zwischen Absolventinnen und Absolventen derselben Hochschule bereits unmittelbar nach ihrem Abschluss ein „gender pay gap“ von knapp $8 \%$ besteht. Aufgrund unseres Datensatzes lässt sich diese Lohndifferenz nicht direkt durch die Ausbildung, die Wahl typischer Frauenberufe, Teilzeitarbeit oder Erwerbsunterbrechungen erklären Faktoren wie Diskriminierung und das berufliche Selbstverständnis von Frauen sowie die möglicherweise antizipierte Vereinbarkeit von Beruf und Familie müssen zur Erklärung herangezogen werden.

Um Frauen diese Vereinbarkeit von Beruf und Familie zu ermöglichen, ist es unabdingbar, dass die Betreuungsmöglichkeiten für Kinder ausgebaut werden. Für eine Verringerung der Erwerbsunterbrechungen, die bei Frauen zu großen Gehaltseinbußen und häufig einem Stillstand oder sogar Rückschritt auf der Karriereleiter führen, ist ein weiterer Ausbau der Krippen- und Kleinkindbetreuung zwingend. Aber auch im Kindergartenund Schulalter brauchen Frauen Betreu- ungsangebote, die mit ihren Arbeitszeiten vereinbar sind. Dieses macht Ganztagskindergartenplätze und Hortangebote in der Schule sowie den Ausbau von Ganztagsschulen notwendig, um Frauen die Gelegenheit zu bieten, Vollzeitbeschäftigungen anzunehmen. Wenn Frauen nicht mehr auf ein Angebot von Teilzeitstellen seitens der Arbeitgeber angewiesen sind, wird auch ihre Selbstselektion auf Stellen, wo diese womöglich zuungunsten des Jahresgehaltes-zukünftig möglich werden, wegfallen.

Auch eine veränderte Rolle der Väter könnte dazu beitragen, dass die Diskriminierung oder auch die Selbstselektion von Frauen verringert wird. Die Aussicht, dass es in Zukunft nicht nur die Frauen sind, die Erwerbsunterbrechungen und Teilzeit aus familiären Gründen in Anspruch nehmen, könnte die Karrierechancen von Frauen deutlich verbessern. Dazu kann die Politik einerseits finanzielle Anreize schaffen, andererseits müssten sich dazu auch die tradierten Rollenverständnisse von Frauen und Männern verändern.

Neben allen notwendigen Anstrengungen der Politik zur Beseitigung der Diskriminierung von Frauen sollten diese selbst auch versuchen, einen Teil des „gender pay gap“ für sich zu eliminieren. Frauen haben im Beruf häufig zu wenig Selbstbewusstsein, trauen sich Führungsaufgaben oder Managementaufgaben weniger zu als Männer mit der gleichen Ausbildung (Dilk/Littger 2009). Frauen brauchen sowohl weibliche Vorbilder, die ihnen vorleben, dass auch Frauen Spitzenpositionen ausfüllen können, als auch eine in den Curricula der Hochschulen integrierte Karriereförderung. An Hochschulen eingerichtete Alumni-Netzwerke könnten gleichzeitig die Vernetzung von Frauen untereinander erleichtern, wie auch Mentoring-Programme anbieten, die der Karriereförderung - vor allem der Studentinnen - dienen. Erste Ansätze zur spezifischen Förderung existieren bereits, sollten jedoch stark ausgebaut und in ihrer Wirkung auf reale Lohnunterschiede überprüft werden. 
Achatz, J./Gartner, H./Glück, T. (2004): Bonus oder Bias? Mechanismen geschlechtsspezifischer Entlohnung, IAB Discussion Paper 2, Nürnberg Allmendinger, J./Dressel, K.,/Ebner, C. (2006): Zum Verhältnis von Demografie, Qualifikation und Frauenerwerbstätigkeit, in: Ministerium für Generationen, Familie, Frauen und Integration des Landes Nordrhein-Westfalen (Hrsg.): Demografischer Wandel. Die Stadt, die Frauen und die Zukunft, Düsseldorf, S. 161-172

Arrow, K. J. (1974): Limited Knowledge and Economic Analysis, in: American Economic Review 1, S. 1-10

Becker, G. S. (1971): The Economics of Discrimination, Chicago Becker, G. S. (1993): Human Capital, New York

Bischoff, S. (1990): Frauen zwischen Macht und Mann. Männer in der Defensive - Führungskräfte in Zeiten des Umbruchs, Reinbek Bispinck, R./Dribbusch, H./Öz, F. (2008): Geschlechtsspezifische Lohndifferenzen nach dem Berufsstart und in der ersten Berufsphase. Eine Analyse von Einkommensdaten auf Basis der WSI-LohnSpiegelDatenbank in Deutschland und im europäischen Vergleich, Berlin Blau, F. D./Kahn, L. M. (2003): Understanding International Differences in the Gender Pay Gap, in: Journal of Labor Economics 1, S. 106-144 Blau, F. D./Kahn, L. M. (2006): The U.S. Gender Pay Gap in the 1990s: slowing Convergence, in: Industrial and Labor Relations Review 1, S. 45-66 Blinder, A. S. (1973): Wage Discrimination: Reduced Form and Structural Estimates, in: Journal of Human Resources 4, S. 436-455 Bundesministerium für Familie, Senioren, Frauen und Jugend (BMFSFJ) (2008): Entgeltungleichheit zwischen Frauen und Männern, Einstellungen, Erfahrungen und Forderungen der Bevölkerung zum "gender pay gap“, Berlin

Bundesministerium für Familie, Senioren, Frauen und Jugend (BMFSFJ) (2009): Entgeltungleichheit zwischen Frauen und Männern, Berlin Brader, D./Lewerenz, J. (2006): Frauen in Führungspositionen - An der Spitze ist die Luft dünn, IAB Kurzbericht 2, Nürnberg

Budig, M.J. /England, P. (2001): 'The wage penalty for motherhood', in: American Sociological Review 2, S. 204-225

Busch, A./Holst, E. (2008): Gender Pay Gap Lower in Large Cities than in Rural Areas, in: DIW-Wochenbericht 6, Berlin, S. 36-41

Dilk, A./Littger, H. (2009): Zierfische machen keine Karriere, in: managerSeminare 137, S. 46-50

Doughty, S. (2008): Women's pay gap is blamed on lifestyle choice, in: Daily Mail vom 21.10.

England, P. (1982): The failure of human capital theory to explain occupational sex segregation, in: Journal of Human Resources 3, S. 358-370 England, P./Farkas, G./Stanek Kilbourne, B./Dou, T. (1988): Explaining occupational sex segregation and wages: Findings from a model with fixed effects, in: American Sociological Review 4, S. 544-558 Eurostat (2008): Europa in Zahlen, Jahrbuch 2008, Brüssel Fitzenberger, B./Wunderlich G. (2002): Gender Wage Differences in West Germany: A Cohort Analysis, in: German Economic Review 3. S. 379-414

Fitzenberger, B./Kunze, A. (2005): Vocational Training and Gender: Wages and Occupational Mobility among Young Workers, in: Oxford Review of Economic Policy 3, S. 392-415

Hinz, T./Gartner, H. (2005): Lohnunterschiede zwischen Frauen und Männern in Branchen, Berufen und Betrieben, in: IAB Discussion Paper 4, Nürnberg

Holst, E. (2003): Einkommensunterschiede zwischen Frauen und Männern nehmen in höheren Positionen zu, in: WSI-Mitteilungen 4, S. 243-250
Holst, E. (2006): Women in Managerial Positions in Europe: Focus on Germany, in: Management Revue 2, S. 122-142

Holst, E./Busch, A.,/Fietze, S., Schäfer, A./Schmidt, T./Tobsch, V./ Tucci, I. (2009): Führungskräftemonitor 2001-2006, Bd. 7, BadenBaden

Kleinert, C. (2006): Frauen in Führungspositionen: Karriere mit Hindernissen; in: IAB Kurzbericht 9, Nürnberg

Krell, G. (2010): Führungspositionen, in: Projektgruppe GiB (2010):

Geschlechterungleichheiten im Betrieb, Berlin, S. 423-484

McPherson, M./Smith-Lovin, L./Cook, J. M. (2001): Birds of a

Feather: Homophily in Social Networks, in: Annual Review of Sociology, S. 415-444

Mincer, J. (1970): The Distribution of Labor Incomes: A Survey - With Special References to the Human Capital Approach, in: Journal of Economic Literature 1, S. 1-26

Minks, K. H. (2001): Ingenieure und Naturwissenschaftlerinnen - neue Chancen zwischen Industrie und Dienstleistungsgesellschaft. Ergebnisse einer Längsschnittuntersuchung zur beruflichen Integration von Frauen aus technischen und naturwissenschaftlichen Studiengängen, Hannover Neumark, D. M. (1996): Sex Discrimination in Restaurant Hiring - an Audit Study, in: Quarterly Journal of Economics 3, S. 915-941

Nivorozhkina, L./Nivorozhkin, A. (2008): The Wage Costs of Motherhood. Which mothers are better off and why, in: IAB Discussion Paper 26, Nürnberg

Oaxaca, R. L. (1973): Male-Female Wage Differentials in Urban Labor Markets, in: International Economic Review 3, S. 693-709

Olsen, W.,/Walby, S. (2004): Modelling Gender Pay Gaps, in: EOC Working Paper Series 17, Manchester

Phelps, E. S. (1972): The Statistical Theory of Racism and Sexism, in: American Economic Review 4, S. 659-666

Plantega, J./Remery, C. (2006): The gender pay gap - Origins and policy responses, Brüssel

Polachek, S. W. (1981): Occupational Self-Selection: A Human Capital Approach to Sex Differences in Occupational Structure, in: Review of Economics and Statistics 1, S. 60-69

Ruppert, A./Voigt, M. (2009): Gehalt und Aufstieg: Mythen - Fakten Modelle erfolgreichen Verhandelns, Aachen

Spiegel-Online (2007): Frauen sind vorsichtiger - und verdienen weniger, http://www.spiegel.de/unispiegel/jobundberuf/0,1518,515718,00.html Statistisches Bundesamt Deutschland (2008a): Frauen erreichen $24 \%$ der Habilitationen 2007, Pressemitteilung 220 vom 18.06.

Statistisches Bundesamt Deutschland (2008b): Studierende an Hochschulen - Wintersemester 2007/2008 - Fachserie 11 Reihe 4.1, Wiesbaden

Statistisches Bundesamt Deutschland (2009): Frauenanteil bei Promotionen steigt auf $42 \%$, Pressemitteilung 266 vom 16.07.

Strunk, G./Hermann, A./Praschak, S. (2005): Eine Frau muss ein Mann sein, um Karriere zu machen, in: Mayrhofer, W./Meyer, M./Steyrer, J. (Hrsg.): Macht? Erfolg? Reich? Glücklich? Einflussfaktoren auf Karrieren, Wien, S. 211-242

Topf, C. (2005): Rhetorik für freche Frauen, München

Wanger, S. (2005): Frauen am Arbeitsmarkt - Beschäftigungsgewinne sind nur die halbe Wahrheit, IAB Kurzbericht 22, Nürnberg WSI-Frauenlohnspiegel (2007): Frauen verdienen 22 Prozent weniger als Männer, Pressemitteilung vom 07.03., online: http://www.boeckler. de/320_85262.html 